\title{
A Self-Balancing Direct-Current Bridge for Accurate Bolometric Power Measurements
}

\author{
Glenn F. Engen
}

\begin{abstract}
Until recently, the most accurate microwave power measurements of the bolometric type have required the use of a manual d-c bridge. A self-balancing d-c bridge has been developed that preserves the inherent accuracy of the manual bridge, extends the dynamic range of operation, and greatly simplifies the operating procedure. A general description of the equipment and operating techniques is given, followed by a comprehensive survey of the sources of error accompanying the method and the accuracy achieved.
\end{abstract}

\section{Introduction}

A large amount of the research effort expended in the field of low level microwave power measurement in the past few years has been directed toward a determination of bolometer-mount efficiencies, or evaluation of the validity of the $r-f-d-c$ substitution principle. ${ }^{1,2,3,4}$ Until recently the only accessory instrument of sufficient accuracy to utilize the results of these investigations has been a manually operated d-c Wheatstone bolometer bridge. The use of this device is, however, both tedious and time consuming; the dynamic range over which the desired accuracy is achieved is rather small, and the danger of barretter burnout due to a misstep on the part of the operator is ever present.

A self-balancing d-c bolometer bridge has been developed at the NBS Boulder Laboratories, which to a large measure relieves these problems, while preserving the accuracy and extending the dynamic range of operation. To date, the technique has been employed primarily with barretter-type elements, although on the basis of preliminary results, the technique should prove equally useful with thermistors.

\section{Manual Bridge}

One of the simplest forms of the manual bridge previously employed at the National Bureau of Standards is shown in figure 1 . The $r-f$ power $^{5}$ is given by

$$
P_{r f}=\frac{r_{0}}{4}\left(i_{1}^{2}-i_{2}^{2}\right)
$$

where $i_{1}$ and $i_{2}$ are the total currents required to balance the bridge without and with $\mathrm{r}-\mathrm{f}$ power present, respectively. A precision potentiometerstandard resistor combination is used to measure $i_{1}$ and $i_{2}$. If it is assumed that this measurement

R. W. Beatty and Frank Reggia, An improved method of measuring efficiencies of ultra-high-frequency and microwave bolometer mounts, J. Research NBS 54, $321(1955)$ RP2594.

${ }^{2}$ A. C. Macpherson and D. M. Kerns, A microwave microcalorimeter, Rev. Sci. Instr. 26, 27 (1955).

${ }^{3}$ H. J. Carlin and Max Sucher, Accuracy of bolometric power measurements,

Proc. Inst. Radio Engrs. 40, 1042 (Sept. 1952).
4 D. M. Kerns, Determination of efficiency of microwave bolometer mounts from impedance data, J. Research NBS 42, 579 (1949) RP1995.

5 More correctly, "the retracted d-c power". contains a random error of \pm 0.005 percent, it can be shown that for a typical bolometer operating at 200 ohms and requiring $8.5 \mathrm{ma}$ of bias $\left(i_{1}=17 \mathrm{ma}\right)$ the error in measuring $P_{r f}$ may be as large as 0.02 , 0.3 , and 3 percent at the 10,1 , and $0.1 \mathrm{mw}$ levels, respectively, if all other sources of error are neglected. The small difference between $i_{1}$ and $i_{2}$ at low levels accounts for the deterioration in accuracy. A variety of alternative techniques have been proposed and employed with some degree of success, but it is not within the scope of this paper to examine the manual technique in detail or to establish the maximum accuracy obtainable. In practice, this is often determined by the stability requirements imposed on the generator and measuring system by the time-consuming nature of the technique, rather than by more fundamental limitations.

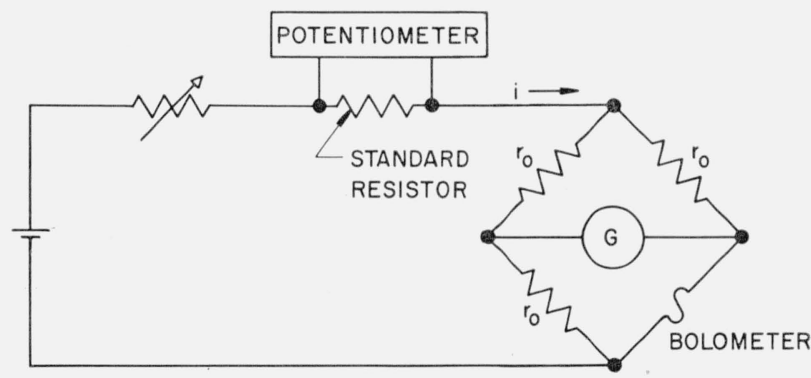

Figure 1. Manual direct-current bolometer bridge.

\section{Self-Balancing Bridge}

The self-balancing d-c bolometer bridge employs a d-c amplifier in a feedback loop to balance the bolometer bridge. The amplifier is connected in such a manner that the bridge unbalance signal is amplified to provide the bridge current (fig. 2). The use of this self-balancing circuit eliminates many of the time-consuming features of the technique, but the power-measurement problem is essentially the same. This problem, at low power levels, is basically one of trying to accurately measure a small change in a comparatively large d-c power. 


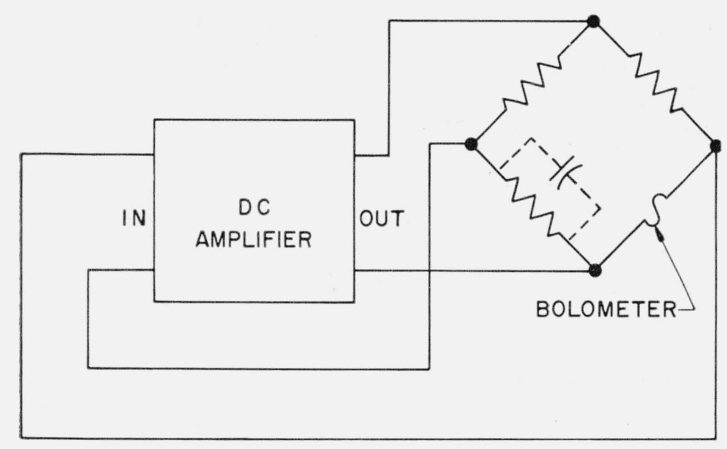

Figure 2. Basic circuit of self-balancing bridge.

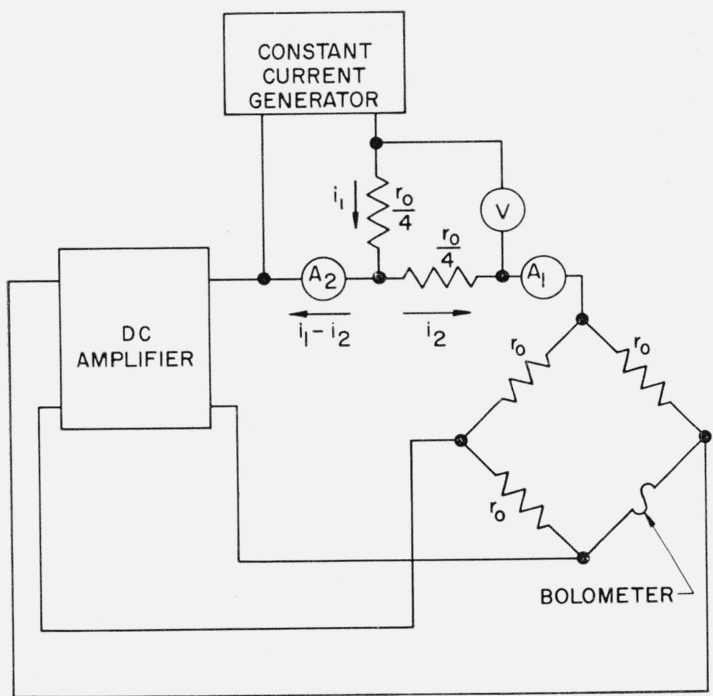

Figure 3. Basic circuit for the determination of $\Delta i$ and power.

The formula for the r-f power may be rewritten

$$
\begin{aligned}
P_{r f} & =\frac{r_{0}}{4}\left(i_{1}+i_{2}\right)\left(i_{1}-i_{2}\right) \\
& =\frac{r_{0}}{4}\left(2 i_{1}-\Delta i\right) \Delta i,
\end{aligned}
$$

where $\Delta i=i_{1}-i_{2}$.

The difficult factor to measure accurately is $\Delta i$. A constant-current generator of high stability has been developed for use in conjunction with a differential current-measuring technique, which enables a direct and accurate determination of $\Delta i$. The circuit is illustrated in figure 3 and operates as follows: First, with no microwave power present in the bolometer, $i_{1}$ is determined by ammeter $A_{1}$, and the output of the constant-current generator is adjusted with the aid of ammeter $A_{2}$ to equal $i_{1}$ (ammeter $A_{2}$ reads zero). Then, with microwave power applied, $A_{2}$ will read $\Delta i$ directly, and the power may be determined from eq (3). In practice, a potenti- ometer and standard resistor are employed in place of $A_{1}$ and $A_{2}$ to achieve high accuracy.

Alternatively, a voltmeter, $V$, connected as shown, will read $\left(r_{0} / 4\right)\left(i_{1}+i_{2}\right)$, and the power is given by the product of $V$ and $A_{2}$. These operations may also be combined in a single instrument of the dynamometer type to achieve a direct reading of the power.

The degree of success obtained with this technique is, of course, contingent upon the degree of stability achieved in the constant-current generator. In this case the change in generator output for a complete cutoff of bridge current is less than 1 part in $10^{5}$, resulting in a negligible error from this source.

\section{Performance}

It is shown in a following section that the over-all accuracy in power measurement achieved, using a potentiometer to measure the current, is about 0.1 percent, whereas substitution of a suitable dynamometer to achieve direct reading is expected to yield an accuracy of about 0.3 percent. The instrument thus provides an increase in accuracy of 10 to 50 times that provided by the commercially avaiablel audiobridges. Other advantages over the tylpica audiobridge include: (1) Comparatively simple techniques are available for establishing, verifying, and monitoring the specified accuracy. (For example, the bridge balance may be monitored by a suitable galvanometer.) (2) The performance is, to a large degree, independent of the nominal deterioriation of the vacuum tubes and other electronic components. (3) The elimination of the audio bias power avoids its interaction with the pulse-repetition frequency in the measurement of pulse power.

At low power levels the $\mathrm{r}-\mathrm{f}$ power is approximately proportional to $\Delta i$ (eq 3), and the low-level performance may be displayed by recording this current with the $r-f$ power alternately on and off. The system response to a $1-\mu \mathrm{W}$ signal is given in figure 4 .

Barring failure of the electronic components, the self-balancing operation eliminates the danger of bolometer burnout due to excessive bias current, whereas protection against an $r-f$ overload may also

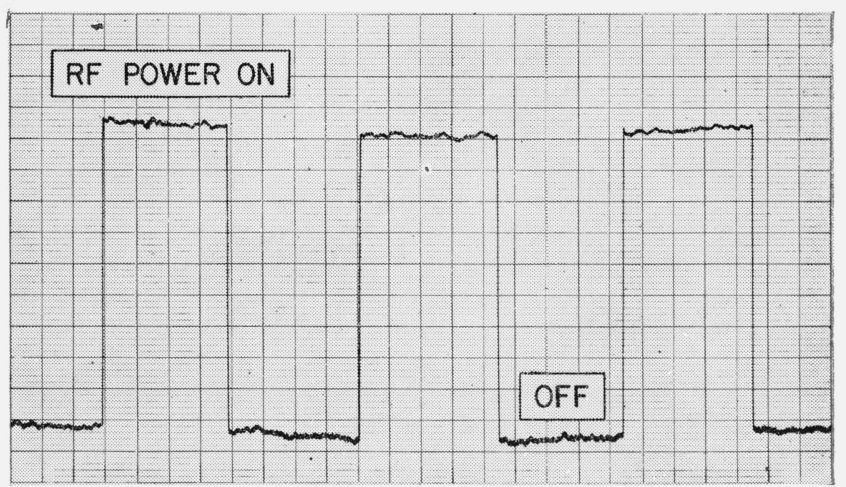

Figure 4. Bridge response to a 1-microwatt signal. (Radiofrequency on and off for 1-minute intervals) 

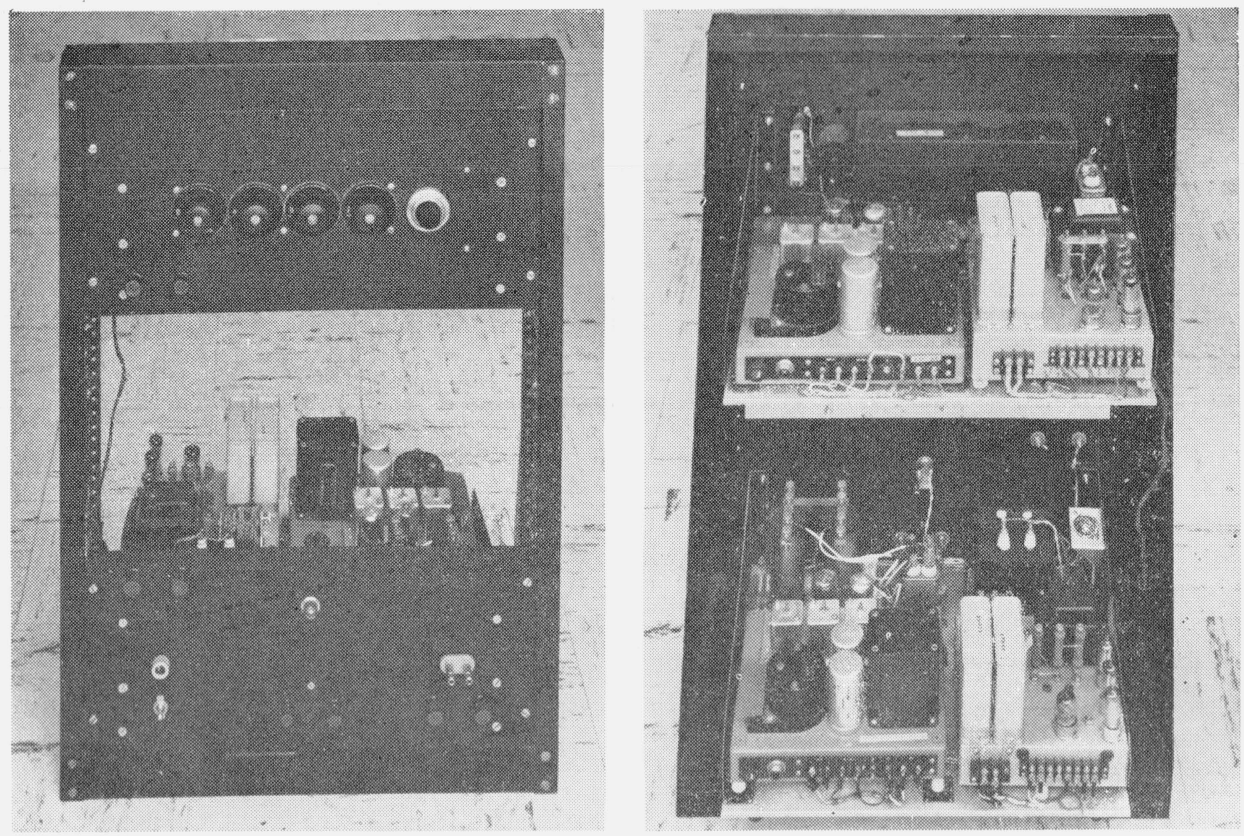

Figure 5. Prototype model of self-balancing bridge, front and rear views.

be provided through use of a suitable relay to turn off the r-f source when the power input to the bolometer exceeds a prescribed amount.

The d-c amplifier employed in this application is a commercially available instrument of exceptional gain, zero stability, and phase response, which was modified by the addition of a cathode follower to provide the required output current. The current amplification of the amplifier, as thus modified, is approximately 100,000 . Compensation for the thermal lag of the bolometer is provided in the feedback loop by means of a condenser, as indicated in figure 2, but the achievement of stable operation with this large amplification is primarily a tribute to the amplifier phase response. The constant-current generator employs a second amplifier of the same type, which, with modification, maintains a constantoutput current by reference against a mercury cell. A prototype model of this equipment is shown in figure 5 .

\section{Analysis of Errors}

The practical problems involved in making an accurate determination of the power, using eq (1) to (3), have been noted. But use of these formulas implies that the bridge is exactiy balanced, the bridge arms are equal to $r_{0}$, etc. It is shown in the appendix that a more complete expression for the power is

$$
\begin{aligned}
P_{r f} \simeq & \frac{r_{0}}{4}\left(i_{b 1}^{2}-i_{b 2}^{2}\right)\left[1+Z-\frac{r_{l}}{r_{0}}+\frac{2 e}{r_{0}\left(i_{b 1}+i_{b 2}\right)}\left(1+\frac{8}{\gamma i_{b 1} i_{b 2}}\right)\right. \\
& \left.+\frac{16(1+g)}{\gamma i_{b 2}\left(i_{b 1}+i_{b 2}\right)}\left(\frac{1}{\alpha}-\frac{i_{g 1}}{i_{b 1}}\right)-\frac{2 g}{\left(i_{b 1}+i_{b 2}\right)}\left(\frac{i_{b 1}}{\alpha}+i_{g 2}\right)\right],
\end{aligned}
$$

where

$\alpha=\left(i_{b 1}-i_{b 2}\right) /\left(i_{g 1}-i_{g 2}\right)=$ current amplification of amplifier

$Z=$ fractional deviation of the lower bridge arm from the value $r_{0}$

$r_{l}=$ resistance of bolometer leads

$e=$ thermal (or other) emf in amplifier input

$i_{b}=$ total bridge current

$i_{g}=$ amplifier input current

$g r_{0}=$ amplifier input resistance

$\gamma=$ "ohms per watt" bolometer coefficient.

For a typical bolometer operating in the 0 to 10 mw region, the following values may be assigned:

$$
\begin{aligned}
r_{0} & =200 \mathrm{ohms} \\
\gamma & =4.5 \mathrm{ohms} / \mathrm{mw} \\
i_{b 1} & =17 \mathrm{ma} \\
i_{b 2} & >8 \mathrm{ma}
\end{aligned}
$$

Substituting these values in the above expression yields the result

$$
\begin{aligned}
P_{r f} \simeq \frac{r_{0}}{4}\left(i_{b 1}^{2}-i_{b 2}^{2}\right)\left[1+Z-\frac{r_{l}}{r_{0}}+5.6 e+18\right. & (1+g)\left(\frac{1}{\alpha}-\frac{i_{g 1}}{i_{b 1}}\right) \\
& \left.-80 g\left(\frac{i_{b 1}}{\alpha}+i_{g 2}\right)\right],
\end{aligned}
$$

where the last three terms in the bracket reflect the inability of the d-c amplifier to balance the bridge exactly.

The characteristics of the d-c amplifier employed in this application are such that the following values 
obtain:

$$
g=1 / 4 \quad \begin{gathered}
\text { (amplifier input resistance } 50 \\
\text { ohms) }
\end{gathered}
$$

$$
\alpha>10^{5}
$$

(current amplification)

$$
\left.\begin{array}{l}
0<i_{g 1} / i_{b 1}<2 \times 10^{-5} \\
0<i_{g 2}<10^{-7} \\
|e|<10^{-}
\end{array}\right\} \text {(amplifier zero stability). }
$$

Substituting these values in the last three terms gives a value for their sum of about 0.0002 .

The bolometer lead resistance can be measured with little effort and the correction applied, so the error from this source is negligible, whereas the value of $|Z|$ can ordinarily be held to 0.0001 or less.

Finally, it is possible to measure current by means of a precision potentiometer-standard resistor combination to an accuracy of a few parts in $10^{4}$; thus the first factor in eq (4) may be determined by the technique discussed earlier to an accuracy of about 0.06 percent.

In summary, the total limits of error are as follows:

Deviation of bridge arms from value $r_{0} \ldots$

Failure of bridge to be exactly

balanced . . . 02 percent.

Measurement of $i_{b 1}+i_{b 2} \ldots \ldots .03$ percent.

Measurement of $i_{b 1}-i_{b 2 \ldots} \ldots \ldots .03$ percent.

Bolometer lead resistance _.... .0 .0 percent.

Amplifier noise and instability (experimentally observed) -. Total

$0.1 \mu \mathrm{W}$. 09 percent $+0.1 \mu \mathrm{w}$.

\section{Appendix}

From figure $6^{\circ}$ the power may be expressed in the form

$$
P_{r f}=\frac{r_{0}}{4}\left(i_{b 1}^{2}-i_{b 2}^{2}\right)+f\left(X, Y, Z, i_{g}, e, r_{l}\right)
$$

where $X, Y$, and $Z$ are the deviations of each of the bridge arms from the value $r_{0}, i_{g}$ and $e$ are the detector current and thermal (or other) emf, respectively, and $r_{l}$ the resistance of the bolometer leads.

Assuming a knowledge of the bolometer resistance law, an exact expression for the second term could be derived, but the labor required suggests the following approximate treatment. By hypothesis, the variables $X \ldots r_{l}$ and $f\left(X \ldots r_{l}\right)$ are small, and $f\left(X \ldots r_{l}\right)$ may, to a good approximation, be written as the sum of the first-order corrections for each of these quantities, where the correction for each of the variables is determined with the other variables equal to zero.

Application of this technique to the circuit in figure 6 yields the following first-order expressions for the d-c power dissipated in the bolometer, and the bolometer resistance

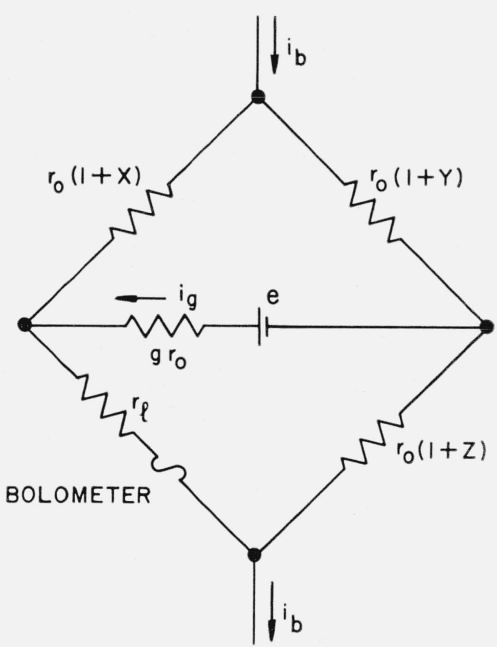

Figure 6. Bridge circuit used in the evaluation of errors.

$$
\begin{gathered}
P_{d c} \cong \frac{i_{b}^{2} r_{0}}{4}\left[1+Z-\frac{r_{l}}{r_{0}}+\frac{2 e}{r_{0} i_{b}}-\frac{2 g i_{g}}{i_{b}}\right], \\
r \cong r_{0}\left[1+x-y+Z-\frac{r_{l}}{r_{0}}+\frac{4 e}{i_{b} r_{o}}-\frac{4(1+g) i_{g}}{i_{b}}\right] .
\end{gathered}
$$

The failure of the expression for power to have a first-order dependence on $X$ or $Y$ is explained as follows: A prescribed, small value for $X$ or $Y$ will cause a first-order variation in bolometer resistance $r$, as indicated in eq (7), but also produces an unequal division of current in the two sides of the bridge. These two sources of error cancel to a first approximation. The only general requirement on the upper bridge arms is that they be equal, and if they have a nominal value of $r_{0}$, it can be shown that they may differ from each other by as much as 2 percent and produce:an error in the power measurement of only 0.01 percent.

At a constant ambient temperature and assuming equivalence of $\mathrm{d}$-c and $\mathrm{r}$-f heating, ${ }^{6}$ the power, $P$, dissipated in the bolometer will be a function of its resistance, $r$ :

$$
P=f(r) .
$$

Because the bolometer is nearly a square-law detector, one may in the neighborhood of an arbitrarily selected operating resistance $r_{0}$, write

$$
P \cong P_{0}+\frac{\partial P}{\partial r}\left(r-r_{0}\right)
$$

where $P_{0}$ is the power required to bring the bolometer to the resistance $r_{0}$ at the given ambient temperat ure, $P$ is the sum of the $\mathrm{d}-\mathrm{c}$ and $\mathrm{r}-\mathrm{f}$ power, $P=P_{d c}+P_{r f}$, and $\partial P / \partial r$ is the reciprocial of the "ohms-per-watt"

6 The r-f-d-c substitution error is assumed to be negligible in this discussion. For a treatment of this problem, see footnotes 2 and 3 . 
bolometer coefficient, i. e., $(\partial P / \partial r)=(1 / \gamma)$. Then

$$
P_{d c}+P_{r f} \simeq P_{0}+\frac{1}{\gamma}\left(r-r_{0}\right) .
$$

$P_{0}^{-}$may be determined by measurements in the absence of $\mathrm{r}-\mathrm{f}$ power:

$$
P_{0} \cong P_{d c}-\frac{1}{\gamma}\left(r_{1}-r_{0}\right),
$$

where the subscript 1 has been used to indicate the value of quantities measured in the absence of $r-f$ power, whereas the subscript 2 will indicate values with $\mathrm{r}-\mathrm{f}$ present.

The formula for $r-f$ power is then

$$
P_{r f}=P_{0}-P_{d c}+\frac{1}{\gamma}\left(r_{2}-r_{0}\right)=P_{d c 1}-P_{d c 2}-\frac{1}{\gamma}\left(r_{1}-r_{2}\right) .
$$

In the given application, only $i_{g}$ of the variables $X \ldots r_{l}$ has a functional dependence upon $i_{b}$, or is subject to change with the application of $\mathrm{r}-\mathrm{f}$ power. Substituting eq (6) and (7) in eq (8) gives the desired result

$$
\begin{aligned}
P_{r f} & \simeq \frac{r_{0}}{4}\left(i_{b 1}^{2}-i_{b 2}^{2}\right)\left[1+Z-\frac{r_{l}}{r_{0}}+\frac{2 e}{r_{0}\left(i_{b 1}+i_{b 2}\right)}\left(1+\frac{8}{\gamma i_{b 1} i_{b 2}}\right)\right. \\
& \left.+\frac{16(1+g)}{\gamma i_{b 2}\left(i_{b 1}+i_{b 2}\right)}\left(\frac{1}{\alpha}-\frac{i_{g 1}}{i_{b 1}}\right)-\frac{2 g}{\left(i_{b 1}+i_{b 2}\right)}\left(\frac{i_{b 1}}{\alpha}+i_{g 2}\right)\right],
\end{aligned}
$$

where

$$
\alpha=\frac{i_{b 1}-i_{b 2}}{i_{g 1}-i_{g 2}}
$$

The author extends his thanks to R. W. Beatty and John E. Lauer for their valuable criticisms and suggestions in the preparation of this paper, and to Ray R. Rumfelt for his assistance in the design and construction of this equipment.

Boulder, Colo., November 28, 1956. 